stretching until a good-sized pouch of the posterior wall of the stomach can be brought through it. Search is now made for the junction of the duodenum and jejunum, close to the root of the transverse mesocolon and just to the left of the vertebral column. A portion of the stomach, as near as possible to the greater curvature and at the most dependent part of the organ, is now clamped in forceps, the blades of which are covered with rubber tubing, the portion thus seized should give plenty of room for the application of sutures over a length of approximately 3 in. A portion of the jejunum some 12 in. or so from its commencement is emptied of its contents and similarly clamped, and the two clamps are brought side by side and held by an assistant in convenient position for suturing the two viscera together; the clamps not only restrain haemorrhage and the visceral contents from escape, but are very useful means of manipulating the parts in the process of suturing. The loop of jejunum should be so arranged that its contents pass onwards from left to right, and not in the opposite direction. For the purpose of suturing I use a curved needle, about $1 \frac{1}{4}$ in. long, with a slight cutting edgethat is, not a rounded needle-and a continuous thread suture of No. 70 calibre, 32 in. long. In the first or exterior row of suturing the needle picks up only the peritoneal and muscular coats, and it is entered about five times in 2 in.; each stitch is drawn fairly tight, and held by an assistant, and avoids all risible blood vessels. The line of suturing is semilunar, and should extend for about 3 in., a knot being tied at the first point of suture, and an end of 2 or 3 in. in length left. The suture is knotted again when the semilunar line is complete, and the end is left threaded with its needle for future use. The two viscera are now opened for a length of nearly 2 in., and some of the redundant mucous membrane is removed from each, so as to leave the anastomotic opening quite patent. If the clamps have been efficiently applied, there is complete control of visceral contents and of bleeding. The second row of suturing is now made with a similar suture to that used in the first, but now the whole thickness of stomach and bowel wall is penetrated by the needle, the stitches are rather more frequently inserted and are drawn quite tight; there is no interruption in the continuity of this line, which runs right round the anastomotic opening. When this is finished the clamps are removed, and the first row of suturing is completed with the suture previously laid on one side. Each row of sutures has a definite purpose: the inner row unites the margin of the opening, and at the same time is haemostatic; the second row is put in to segregate the first.

Whilst I prefer this method of performing the operation to any other, I recognize that occasionally the use of the Murphy button is distinctly advantageous, chiefly where from presence of adhesions it is difficult to expose a sufficient area of the stomach wall to ensure a safe suturing. I have had two cases of this kind where gastro-jejunostomy was required for pyloric stenosis aiter the patients had recovered from perforation of the stomach. Surgeons who are only called upon to perform this anastomosis occasionally may well find that the safest method is to employ the Murphy-button, being prepared, like myself, with increasing experience to discard it for suture.

What is the mortality of the fairly the innocent cases must be separated from the malignant ones, and as I have not treated of malignant disease in my remarks hitherto, what I am now about to say applies only to innocent strictures, and in these I think the deathrate will prove to be, in the hands of those accustomed to the operation, about 5 per cent. There is no doubt whatever, though, that the extent of the mortality will be materially influenced by the time at which operation is performed. Given patients in moderately good condition, not worn out by years of malnutrition and extreme loss of weight, I think the mortality of the operation will be less than 5 per cent.; but if the cases are such as may be described as "late" ones, then the mortality will be distinctly higher than the figure at which I have placed it. Another important question is, To what extent are the patients who survive operation benefited ? Happily, the answer is that they are enormously benefited; they gain weight, they regain the power of work, and have a power of digestion which they have been strangers to for years. They are able generally to take an ordinary mixed diet without pain or any other inconvenience. At one time the fear of contraction of the new opening loomed large, but time has proved this to be unfounded; at all events, contraction takes place very rarely, if at all, and I believe this to be true whether the operation is by suture or by some of the other methods in vogue.

After-treatment.

For twelve hours nothing is given by the mouth, then small sips of water are permitted, and at the end of thirty-six hours $\zeta$ ss of diluted or of peptonized milk is given hourly. The quantity of milk is increased every few hours, until at the end of the fourth day $1 \frac{1}{2}$ pints of milk are being disposed of. Benger's food, custard, and similar foods are now added, and about a week after the operation minced chicken or mutton is permitted. At the end of a fortnight a varied diet of toast, vegetables, milk, meat, etc., is being consumed. For the first few days after operation nutrient enemata are administered every four to six hours. Some vomiting occurs in a majority of cases, probably due as a rule to the anaesthetic, but speedily subsides. Some patients do not vomit at all, others a good deal, owing to regurgitation of the duodenal contents into the stomach. This regurgitation may occasionally give rise to real anxiety, but I think I may say that with increasing experience of the operation, regurgitant vomiting becomes less common and of less importance.

The patients should be kept in bed for at least three weeks after operation, and an extra week of recumbency is often advantageous. At least another month is required for the recovery of lost weight and condition before work is attempted, and if circumstances allow, this period of convalescence should be prolonged.

\section{DUODENAL ULCER AND ITS TREATMENT.}

BY D'ARCY POWER, F.R.C.S.ENG., Surgeon, St. Bartholomew's Hospital.

THE subject of duodenal ulcer has not yet received adequate attention, though its onset is severe and the sequelae may be dangerous. It is usually thought to be of rare occurrence, but during the last few years I have myself operated upon seven cases, and I have had the opportunity of seeing others which have been under the care of my colleagues at St. Bartholomew's Hospital. From these cases I have learnt so much that I propose to bring some of the facts before you this evening that you may the better recognize the condition and give it appropriate treatment.

I have thrown my seven cases into a tabular form, and you will see that they group themselves sharply into two classes, those in which the duodenal ulcer had perforated, and those where there was no perforation. The cases which perforated required immediate surgical treatment, and were sutured with more or less success. Those which did not perforate were either treated at once on account of haemorrhage, or came for treatment many years afterwards in consequence of duodenal narrowing due to cicatrization of the ulcer. The non-perforating cases were treated by retro-colic gastrojejunostomy.

I do not want to weary you with lengthy and uninteresting details of the cases, so perhaps I may be permitted to draw composite pictures of the two classes of cases, premising that every case of duodenal ulcer which has come under my observation has occurred in a man, so that duodenal ulceration is probably more common in men than women.

The subject of a perforating duodenal ulcer is usually a man in the prime of life who has hitherto considered himself in perfect health, or who has suffered, if at all, from only the slightest symptoms of indigestion. Without warning he is suddenly seized with a stomachache of such severity that he becomes collapsed and sends at once for assistance. He may vomit, but from the onset of the pain he passes neither flatus nor faeces. Examination within an hour or two of the attack shows him lying on his back, afraid to move, his breathing shallow and very rapid ; his pulse small, regular, and quick, but not nearly so much accelerated as his respiration. Temperature normal. He looks pinched and anxious. The patient cannot localize his pain, but complains that it is worse along the upper half and down the right side of the abdomen. The abdomen is not distended and is not motionless, though it moves less freely during respiration than it should do. It is held rigid, and the muscles on the right side are somewhat more tonically contracted than those on the left. It is everywhere tender and tympanitic. The area of liver dullness may or may not be altered, and sometimes 
there is a point of maximum tenderness in the right bypochondrium.

If no operation be performed the pain becomes less acute but even more generalized than it was at first. Later still it is localized in the right iliac region, which may become especially tender, full, and motionless, whilst the rest of the abdomen moves during respiration. More usually, however, the abdomen becomes uniformly distended, and the patient, who has rallied from the initial shock, again becomes collapsed, his pulse rapidly quickens, and he dies of acute peritonitis.

Now look at a case where there has been a duodenal ulcer which did not perforate. It is even more interesting than the last, for the diagnosis is more difficult and the sequelae are more remote. The patient is an older man, perhaps between 50 and 60 . Thin and haggard, he tells you that he is a martyr to indigestion, and that for months past he has suffered trocious pain in his stomach, which is relieved by vomiting He has dieted himself in every possible manner, he has made all kinds of local applications to his abdomen, he has visited all sorts of watering places, and he has gone in vain from one physician to another seeking a cure. Examination shows him to be a mere bag of bones, badly constipated, with cold extremities, and a listless, dejected aspect. His abdomen is loose, the subcutaneous veins may be enlarged, and there is visible peristalsis from left to right in the epigastric region. Percussion tells you that the stomach is greatly dilated, and it is not very unusual to feel a tumour in the neighbourhood of the pylorus. For a moment you think of cancer of the pylorus or gall bladder, and you question the patient a little more closely. $\mathrm{He}$ is sure that he has been suffering for years, for so long, in fact, that he hardly recollects the beginning of his trouble. A few well-directed inquiries may elicit that twenty-five or thirty years ago, when he was a young man, he once or twice brought up a large quantity of blood without serious pain or discomfort, or that he had an illness which no one seemed to know much about. He was treated for gall stones, or appendicitis, or simply for "liver." The attack was painful, and kept him in bed, but the exact details have passed from his mind, and for some years he was as healthy a man as ever.

This is a case of duodenal obstruction resulting from cicatrization of an old ulcer, the irritation of which has caused inflammatory thickening in the surrounding parts. How many patients have been allowed to die of such a condition in the belief that they had malignant disease of the stomach no one can tell. But for such patients a gastro-jejunostomy holds out the prospect of $a$ speedy and perfect cure.

The pictures I have painted for you are composite in the sense that no individual patient presents all the characteristics indicated, though all resemble each other sufficiently closely to enable a diagnosis to be made with comparative ease, as soon as one becomes alive to the occurrence of duodenal ulceration and its sequelae. I shall proceed to examine the signs, symptoms, and treatment, a little more in detail.

The diagnosis of a perforated duodenal ulcer should be easy, but in practice it is often found to be a matter of very great difficulty. The patient is obviously guffering from acute abdominal pain, and the attack began suddenly. The history of previous good health, the fact that the abdomen is moving during respiration, the absence of any great amount of rigidity in the abdominal muscles, or of any point of maximum tenderness, are all misleading, and make it difficult for the surgeon to believe that the patient is suffering from such a dangerous condition. One thinks, indeed, of a perforated or leaking gastric ulcer, but the symptoms are much less characteristic, and it is not until one or two similar cases have come under one's observation, that the occurrence of a perforated gastric ulcer is thought of. The diagnosis, therefore, is too often left in abeyance, in the hope that a few hours' delay will render the signs and symptoms more definite. Such advice is likely to prove fatal, for instead of making the diagnosis clearer time only renders it more obscure. The slight clues which could be picked up shortly after the onset, are soon masked by the peritonitis which follows. Delay not only allows the peritonitis time to develop, but it permits the extravasated contents of the alimentary canal to gain access to the innermost recesses of the peritoneum, so that a subphrenic, pelvic, or iliac abscess may still further complicate a condition which is well-nigh desperate. When I am called to a patient who has been suddenly seized with intenge abdominal pain, without much history of previous indigestion, I ask that he shall not be given morphine, and that his palse shall be carefully and accurately counted, and recorded every half-hour. If on my arrival the pulse-rate has increased, although the patient has been kept at rest and ree from disturbing influences, I have no hesitation in advising an immediate exploratory operation, even though the objective signs be very slight.

The escape of gas and liquid as soon as the abdomen is opened prove that a perforation has occurred, and the surgeon's first thought will be that he is dealing with a ruptured gastric ulcer. The character of the fluid gives the first indication as to the seat of the perforation. It is limpid or bile-stained, free from smell, alkaline, and quite different from the extravasated contents of the stomach. It is the succus entericus, and is, I presume, the secretion of Brunner's glands. It is very abundant, and comes welling up from the perforated intestine in quite a characteristic manner. The perforation is usually very small, close to the pylorus, and rather at the back of the duodenum, so that it is very awkwardly placed for suture.' It is possible, however, to close it ; one of my patients recovered after suture, and is now doing his usual work more than two years after the operation; and there are doubtless many others on record if one chose to look through the literature of the operation.

If the patient be left without operation the diagnosis, as I have said, becomes still more obscure, and my own experience tells me that a perforated duodenal ulcer may be mistaken for pneumonia, for appendicitis, or for suppurative peritonitis due to causes other than intestinal perforation. The idea of appendicitis is so constantly present to the mind of a surgeon as the cause of acute abdominal trouble that the mistake is excusable, and the more so as the symptoms of perforated duodenal ulcer sometimes closely simulate those of an inflamed appendix. I made the mistake in the case 0 a man of 26, whose duodenal ulcer perforated one evening at 7 o'clock. I did not see him until 10 o'clock next morning, when he localized his pain over the right iliac region, which was tender and full. The abdominal muscles were tense and the abdomen was motionless on the right side, but the reat of the abdomen moved well during respiration. I thought the patient was suffering from an acute appendicitis which had ended in perforation, and I opened the abdomen in the right iliac region. Gas issued from the wound as soon as the peritoneum was divided, and there was a gush of inodorous, alkaline fluid, which was bile-stained. The appendix was normal, but the end of the ileum was inflamed in patches which seemed to correspond with Peyer's patches. I closed the wound, cut down upon the duodenum, found the ulcerated spot, sutured it, and the patient recovered.

In another case where the diagnosis of pneumonia was made the patient was a man of $4 \mathrm{I}$, whose ulcer had perforated at 11 a.m. When he was seen at 2 o'clock he was in obvious pain, breathing shallowly and 60 times a minute His pulse was of fair volume and tension, regular, and 100 a minute. The percussion note of the chest was impaired at the right base, and the entry of air on the right side was weaker than on the left. There were no additional sounds. The abdominal pain was not localized, but the patient complained of it chiefly over the upper half and down the right side. The abdomen was not distended, but it moved very little during respiration. The movements, however, were equal all over, though the abdomen was held a little rigid. It was tender and tympanitic everywhere, except that the liver dullness was present. Nothing abnormal could be felt. I did not see this patient until two o'clock next morning, when his condition had become so serious, and the abdominal symptoms were so marked, that I had no hesitation in opening his abdomen. I found and partially sutired a perforation at the upper and posterior part of the duodenum close to the pylorus, but the patient was in no state to bear a prolonged operation, and he died two hours later.

The lesson I have learnt from these cases of perforation by a duodenal ulcer is to operate early. The perforation often takes place without warning, and it thus occurs in people who are otherwise in excellent health. Such people bear an abdominal exploration very well, and I would much rather open an abdomen needlessly than wait, as was done in the case just narrated, until symptoms of peritonitis made an operation imperative.

The second group of cases, where there is a duodenal ulcer without perforation, is even more interesting than the perforating variety which has been considered. The diagnosis is more difficult, and the sequelae demanding surgical trestment are no less urgent, though they may be more remote. 
As may be gathered from what has been said of perforating ulcers, the symptoms of a duodenal ulcer may be absent or wholly inconspicuous, and in such cases no diagnosis can be made. In some cases the patient complains of a continued pain in the abdomen, which he can neither localize nor account for. He keeps his bed for a few days, and then, feeling better, goes about his business, saying that he has had a bad bilious attack. If he is nervous about himself, or should the pain be severe, he seeks medical advice, and is treated for gall stones, renal colic, or appendicitis. But the exact nature of his illness is not recognized until he brings up a considerable quantity of blood, or has a sharp attack of melaena. Even then the case is probably thought to be one of gastric ulcer, unless an abdominal section is performed, and the stomach is found to be healthy. I do not know how the error can be avoided in the present state of our knowledge, but it is of no consequence, for in the majority of cases the patient recovers from the attack, or, if the bleeding is sufficient to need an exploration, the surgeon performs a gastrojejunostomy, whether the ulcer is in the stomach or the duodenum. I have tried to recognize the condition by the bleeding, by the time of the occurrence of pain after taking food, by the existence of localized tenderness, by the character of the material vomited, and in many other ways, but always without success; for what is true and seems a valuable sign in one patient is perfectly worthless in another. I believe, therefore, that at the present time there is no pathognomonic sign of a non-perforating ulcer of the duodenum.

But if a non-perforating duodenal ulcer offers very few signs by which its presence may be recognized, it may have sequelae of the gravest importance. The ulcer is usually single, small, conical in shape, and is most often situated in the first part of the duodenum close to the pyloric fold. It runs a chronic course, and may either heal and by subsequent cicatrization narrow the duodenum, or, as more frequently happens, adhesions may be formed to surrounding parts. The adhesions contract in course of time, and may so narrow the duodenum as to render it useless for the passage of the contents of the stomach. The adhesions at the same time may involve the liver, the gall bladder, the pancreas, or the great blood vessels, like the abdominal aorta, the hepatic artery, and the portal vein. The patient wastes, his stomach becomes dilated, the enlargement of the superficial abdominal veins shows that the deep venous circulation is impeded, and a swelling is readily detected in the region of the pylorus. Small wonder, therefore, that nearly all these cases are diagnosed as cancer of the pylorus or cancer of the gall bladder. Indeed, it is only of late years that it has been possible to distinguish between these forms of abdominal cancer and the symptoms produced by the cicatrization of $a$ healed duodenal ulcer. The knowledge has been gained by opening the abdomen to perform a gastro-enterostomy for the relief of cancer, and finding, to the satisfaction of patient and surgeon alike, that there was nothing worse than a mass of adhesions, and that the operation of gastro-jejunostomy promised permanent instead of merely temporary relief. Care and attention to details, however, sometimes render it possible to distinguish between cancer and a cicatricial contraction of the duodenum, and in my last case $I$ was able to make a correct diagnosis before I decided on an operation.

Dilatation of the stomach is a necessary consequence of chronic obstruction at or near the pylorus. The symptoms last longer in a non-malignant than in a cancerous constriction, and the dilatation consequently is greater in an innocent than in a malignant stricture of the duodenum. There is often visible peristalsis, which can also be felt, in the upper part of the abdomen. The whole stomach may become so displaced downwards that the lesser curvature lies 2 in., or even 3 in., below the costal margin. A distinct succussion splash can be heard over the dilated stomach, unless the organ has been recently emptied by vomiting. The gas evolved within the stomach by giving the two parts of a seidlitz powder separately may be insufficient to distend fully the flaccid stomach. The patient is thin, his skin is harsh and dry, he is constipated, and passes but little urine. He complains of attacks of vomiting which bear no relation to his meals. The attacks come on every day or two, and are worse at night. Very large quantities of fluid are vomited, and sometimes there are streaks of blood, sometimes there is bile. The stomach seems to be completely emptied, yet in a few hours he again vomits copiously, though he has taken nothing in the interval. The vomit is often very acid, owing to the presence of free hydrochloric acid, and the fluid is clearly the secretion of the gastric and duodenal glands. This secretion should be reabsorbed during the intestinal digestion in a state of health, but no such absorption is possible when the duodenum is constricted, and the patient is constantly draining his lymphatic system, and indirectly his blood, to supply the fluid he vomits. Although the ingestion of food does not produce vomiting, yet the patient curtails his diet as far as possible, because nearly everything he eats causes a sensation of weight and fullness in his stomach. Hiccough is sometimes a marked and very troublesome feature, and the patient becomes so restless and miserable that he cannot long keep still in one position or place.

Gastro-enterostomy yields a most brilliant result in these patients. As soon as the shock of the operation is over, they begin to put on flesh; vomiting and the hiccough which was previously so distressing cease absolutely, the bowels return to their wonted regularity of evacuation. These good results are not merely temporary, as is too often the case when a gastro-jejunostomy has been performed for gastric ulcer. When gastro.jejunostomy has been done for the relief of pain, in a case of gastric ulcer, the pylorus is patent and functional, and there is a marked tendency for the anastomotic opening to close or become so small as to be useless. It often happens, therefore, that the dyspeptic symptoms are relieved; and the patient improves markedly for a time, but within four months of the operation the improvement ceases, and the condition of the patient either remains stationary or actually recedes. When a gastro-jejunostomy has been done for duodenal constriction, the anastomotic opening shows no tendency to close, for the duodenal constriction remains, and the food must necessarily pass through the new opening from the stomach into the intestine. The good effects of the operation, therefore, are permanent. The patient's mind, too, is set at rest by the operation. He is sure to have been told or to assume that he is suffering from cancer of the stomach, and it is a great relief to know that he has only got a mechanical obstruction. He is bound to regulate his diet, and thus he acts in accordance with the precept laid down for us in the Regimen Sanitatis Salerni, our first popular textbook of medicine:

Si tibi deficiant Medici, medici tibi fiant

Haec tria: mens hilaris, requies, moderata diaeta.

which was Englished :

Use three Physicians still : first Dr. Quiet, Next Merryman, then Doctor Diet.

I do not propose to enter upon the broad question of gastroenterostomy and its results this evening. It is sufficient to say now that I prefer the operation of gastro-jejunostomy by direct suture and by the post-colic route. The most satisfactory results from it are obtained in cases of duodenal ulcer; and the least satisfactory when the operation is performed for cancer of the stomach.

I have dealt this evening entirely with duodenal ulcer and its consequences, but I do not wish to leave upon your minds the impression that all or even most of the cases of pyloric obstruction are a consequence of duodenal ulceration. There are many other causes, of which gall stones and cancer are the most common in adults, faulty development and congenitar hypertrophy of the pylorus in infants. My thesis deals only with duodenal ulceration and its consequences.

I have tried to show that:

(a) Duodenal ulcers are not very uncommon.

(b) So far as I have seen them, duodenal ulcers are singl: and are more frequent in men than in women.

(c) Duodenal ulcers may perforate and cause acute symptoms, or they may heal, and by cicatrization lead tosymptoms of chronic duodenal obstruction.

(d) The sequelae of a healed ulcer may be so remote that thesymptoms are mistaken for those due to cancer of the pylorus, and the patient is allowed to drift from bad to worse under the erroneous notion that he is bound to die.

(e) There is no means of recognizing the existence of duodenal ulcer, in a great many cases, until it perforates or until the results of its cicatrization become manifest.

(f) The treatment of duodenal ulceration consists (I) in the direct suture of a perforated ulcer, the prognosis being: less favourable than in similar cases of perforation; (2) the performance of gastro-jejunostomy in cases of dilated stomach due to duodenal constriction, the prognosis being the most favourable of all the conditions for which this operation is performed at the present time. 
A.-Cases of Perforating Duodenal Ulcer Treated by Suture. Case I.-May 23rd, rgor. Male, aged 4r, temperate. No history of previous illness. Sudden pain in the epigastrium at $1 \mathrm{r}$ a.m. Applied for rellef at 2 p.m. When he was first seen his pulse was roo; pain felt in right side; abdomen moving slightly and somewhat rigid. The symptoms were so ill-defined that he was thought to be suffering from pneumonia. At 12.30 midnight his pulse was 120 and his abdomen distended. I saw the patient for the first time at 2 a.m. and opened his abdomen. A punched-out ulcer was found just beyond the pylorus, from which clear fluid kept welling up.

Result.-Death five hours and a half after the operation. Post-mortem cecord : Imperfect closure of the perforation.

CASE II.-July r 3 th, rgor. Male, aged 47, a drunkard. No history of previous illness. Sudden, very severe abdominal pain at 9 a.m., but did not apply for relief until 8.30 p.m. When first seen his pulse was I04; the abdomen was moving during respiration. There was general tenderness, most marked in the epigastric region. Abdomen opened at co.45 p.m. I found a circular and well-defined perforation at the back of the pylorus. Clear fluid was welling up through it.

Result.-Death eight days later. The patient felt so well until twentyfour hours before his death that he was constantly asking when he might get up. Post-mortem record : Subdiaphragmatic abscess ; incomplete closure of the perforation. The ulceration had continued after the sutures had been inserted (? cancerous).

CASE III.-May r8th, rgo2. Male, aged 37, temperate. Had complained for the last six months of stomach-ache half an hour after his meals. He had felt a sudden pain at twelve o'clock mid-day, on the right side beneath his ribs. The pain was so severe that he took a cab to the nearest hospital. On admission the pulse was $=20$, the abdomen was moving freely and equally. I operated at 6 p.m. There was no perforation in the stomach, but a small clean-cut ulcer in the duodenum, from which bile-stained fluid kept pouring out. The aperture was closed, and a counter-incision was made in the right umbar region, through which a drainage tube was passed.

Result.-Death 35 hours after the rupture. Post-mortem record: The perforation was effectively closed, as it was water-tight.

CASE IV.-July 26th, rgo2. Male, aged 26, temperate. Had suffered previous attacks of a similar pain, but none so severe as that which occurred suddenly at 7 p.m. He was admitted to the hospital at $3.50 \mathrm{a.m}$. on July 27 th, but I did not see him until ro a.m. on this day. His pulse was 122 . There was pain over the right iliac region, which was full and tender. The abdomen, except over the tender area, was moving during respiration. I thought he was suffering from an acute inflammation of the appendix, but on opening the abdomen in the lliac region, the appendix was found to be normal. The stomach was intact, but a perforation was discovered on the anterior surface of the duodenum near the pylorus.

Result. - The patient left the hospital cured on September 12 th, 1902. I have seen him on more than one occasion in the last year. He is at work, and says (October $20 \mathrm{th}, 1904$ ) he is able to do his work but has to be careful in his diet.

B.-Cases of Non-perforating Duodfnal Ulcer treated BY GASTRO-JEJUNOSTOMY.

CASE V.-Male, aged $3 \mathrm{r}$, temperate. Was admitted to the hospital with pain in the lower part of his abdomen and vomiting, which had made him an invalid for two months. He retched after food, and vomited at one time "a quart of dark blood, all in lumps," and at another time " a pint of blood." There was no melaena. On admission he had a point of maximum tenderness in each iliac fossa, and a tender point $I$ in. to the right of the umbilicus, which varied somewhat in position. I diagnosed the existence of a duodenal ulcer, and performed a post-colic gastro-jejunostomy on May Igth, 1904

Result. - The patient made a perfectly uneventful recovery, and left the hospital on June 17 th, r904. In November, x904, he is improved but not well.

CASE vI.-Male, aged 69, temperate. Admitted to hospital, August ist, r902, for constant vomiting; a greatly dilated stomach, and a tumour as large as a Tangerine orange in the neighbourhood of the pylorus. The patient had lost mush flesh. I performed a gastro-jejunostomy with McGraw's elastic ligature.

Result.-The patient recovered completely, with increase of weight and cure of dyspepsia. I saw him in July, x9.04, a hale and hearty man of $7 \mathrm{r}$, just starting to Canada.

CASE VII.-Male, aged 48 , temperate. Admitted to hospital on July 3 rst, r904, complaining of intense abdominal pain reliéved by vomiting. He had lost much flesh during the last few months. On admission his stomach was found to be dilated, there was evidence of slight portal obstruction, but no tumour could be felt in the region of the duodenum. I diagnosed the case as one of duodenal constriction, and performed a retro-colic gastro-jejunostomy on August 18t, 1904. On opening the abdomen the duodenum was found to be constricted to the size of a lead pencil, about an inch beyond the pylorus.

Result.-The patient made an uneventful recovery, and left the hospital on August rgth, rgo4. In November he reports himself quite well, returned to his normal weight of 12 st., and walking ro miles a day in comfort.

The Spitting Nuisance in Bunnos Aires.-At a recent meeting of the Municipal Council of Buenos Aires a proposal was submitted by Dr. Conti that owners or tenants of houses and buildings who refused to allow tablets to be put up on the outside forbidding expectoration on the pavements should be fined. The proposal was referred to a committee.

\section{Sotentifio Grants Committee} OF THE Britisb Medical Hssociation.

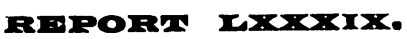

\section{THE NATURE OF THE MALFORMATIONS OF THE RECTUM AND UROGENITAL PASSAGES.}

By F. WOOD-JONES, M.B., B.Sc.Lond., M.R.C.S.EnG.

[THIS paper is a brief summary of the clinical side of a paper on the Development and Musculature of the Visceral Openings of the Hind End, accepted by the London University for the Degree of B.Sc., by Research, October, 1903.]

When the details of human embryology have merely an academic interest they are of little utility in a medical education, and serve no useful purpose in the object of all medical training - practical medicine and surgery.

To be of any value at all to the medical man, embryology must help him to understand how, and why, the adult organs become developed, it must furnish him a ready explanation of the abnormalities and imperfections of development with which he meets, and it should afford some guide to him in the treatment he must undertake for their relief. Regarded in this light, much that is taught of the development of the human body must be admitted to be of little practical value in medicine.

In many cases the teachings of embryology furnish no real explanation of the abnormalities that are met with clinically, and in very few do they afford any guide for the artificial repair of these abnormalities.

The generally accepted view of the development of the human hind end offers no explanation of some of the abnormalities which are commonly found clinically in this situation. The growth of septa and partition walls, which is so often invoked-and often wrongly invoked-as an explanation of various abnormal conditions, is founded on a misinterpretation of developmental changes, and, it would be urged, does not here occur, at any rate in the manner and to the extent that is usually described. It is therefore necessary to inquire if the present account of the development of the human hind end is a correct interpretation of the various stages that have been seen in the human embryo, if some stages have been overlooked, or some stages seen in other animals have been wrongly read into the early chapters of human development. That some stages are wrongly interpreted, and some are wrongly described, and that some abnormal conditions are capable of more practical and more clinically useful explanation, will I believe, be found from a further study of the development of the hind end of the human embryo.

In order to test the shortcomings of the present theory, and to estimate any advantages that some modification of it may possess, it is necessary to see, in outline at any rate, what are the problems that require solution. Most of the malformations of the rectum and urogenital passages that are commonly met with fall into a few well-recognized groups, and it is an outstanding fact in regard to these abnormalities that they are wonderfully constant in their anatomical arrangement. Their very constancy demands that their explanation should be definitely furnished by reference to their development. The visceral openings of the hind end are subject to two classes of malformations. In the first class a terminal septum closes the orifice, and in the second class there may be an actual absence of a portion of the tube which should normally open to the outside at one of the visceral orifices. In the first class come such abnormalities as imperforate anus, imperforate hymen, and urethral septum. In the second class come the more grave conditions of imperforate rectum, atresia vaginae, and atresia urethrae.

The extent of the deformity may not be limited to the actual developmental error, but may be increased and altered by secondary changes that this condition produces, and one such example of this more complex deformity is ectopia vesicae.

The anatomical condition of most of these abnormalities is simple; all those which are included in Class I are alike in possessing a terminal septum, and no discussion of their anatomical features is required. Some of those conditions that have been included in Class II, however, require some 